\title{
Plasmonic semiconductor nanogroove array enhanced broad spectral band millimetre and terahertz wave detection
}

\author{
Jinchao Tong ${ }^{1}$, Fei Suo ${ }^{1}$, Tianning Zhang ${ }^{1}$, Zhiming Huang ${ }^{2}$, Junhao $\mathrm{Chu}^{2}$ and Dao Hua Zhang ${ }^{1}$
}

\begin{abstract}
High-performance uncooled millimetre and terahertz wave detectors are required as a building block for a wide range of applications. The state-of-the-art technologies, however, are plagued by low sensitivity, narrow spectral bandwidth, and complicated architecture. Here, we report semiconductor surface plasmon enhanced high-performance broadband millimetre and terahertz wave detectors which are based on nanogroove InSb array epitaxially grown on GaAs substrate for room temperature operation. By making a nanogroove array in the grown InSb layer, strong millimetre and terahertz wave surface plasmon polaritons can be generated at the InSb-air interfaces, which results in significant improvement in detecting performance. A noise equivalent power (NEP) of $2.2 \times 10^{-14} \mathrm{~W} \mathrm{~Hz}^{-1 / 2}$ or a detectivity $\left(D^{*}\right)$ of $2.7 \times 10^{12} \mathrm{~cm} \mathrm{~Hz}^{1 / 2} \mathrm{~W}^{-1}$ at $1.75 \mathrm{~mm}(0.171 \mathrm{THz})$ is achieved at room temperature. By lowering the temperature to the thermoelectric cooling available $200 \mathrm{~K}$, the corresponding NEP and $D^{*}$ of the nanogroove device can be improved to $3.8 \times 10^{-15} \mathrm{~W} \mathrm{~Hz}^{-1 / 2}$ and $1.6 \times 10^{13} \mathrm{~cm} \mathrm{~Hz}^{1 / 2} \mathrm{~W}^{-1}$, respectively. In addition, such a single device can perform broad spectral band detection from $0.9 \mathrm{~mm}(0.330 \mathrm{THz})$ to $9.4 \mathrm{~mm}(0.032 \mathrm{THz})$. Fast responses of $3.5 \mu \mathrm{s}$ and $780 \mathrm{~ns}$ are achieved at room temperature and $200 \mathrm{~K}$, respectively. Such high-performance millimetre and terahertz wave photodetectors are useful for wide applications such as high capacity communications, walk-through security, biological diagnosis, spectroscopy, and remote sensing. In addition, the integration of plasmonic semiconductor nanostructures paves a way for realizing high performance and multifunctional long-wavelength optoelectrical devices.
\end{abstract}

\section{Introduction}

Millimetre and terahertz wave detectors have a wide range of applications in areas such as communications, security, biological diagnosis, spectroscopy, and remote sensing ${ }^{1-8}$. They are the components that can transform light information loaded by long-wavelength millimetre and terahertz waves into electrical signals. High-performance room temperature detectors with high sensitivity, fast response, broad spectral bandwidth, and possibility to be extended to large

\footnotetext{
Correspondence: Jinchao Tong (jctong@ntu.edu.sg) or

Dao Hua Zhang (edhzhang@ntu.edu.sg)

'School of Electrical and Electronic Engineering, Nanyang Technological University, Nanyang Avenue, 639798 Singapore, Singapore

${ }^{2}$ State Key Laboratory of Infrared Physics, Shanghai Institute of Technical Physics, Chinese Academy of Sciences, 500 Yu Tian Road, 200083 Shanghai, China
}

format arrays are always pursued. They are the building blocks for a wide range of millimetre and terahertz wave related systems, including communication network, deep space exploration equipment, security screening system, spectroscopy system, and material composition inspection. However, conventional efficient photoexcitation in optoelectronic semiconductors seems not applicable due to small quantum energy of millimetre and terahertz waves (1.24 meV@0.3 THz) and strong background thermal disturbances (25.7 meV@300 K). Although Golay cells (such as products from Tydex and Microtech Instr.), pyroelectrics (such as DLATGS, $\mathrm{LiTaO}_{3}$, and products from QMC Instr.), bolometers (such as $\mathrm{HgCdTe}, \mathrm{SiGe}, \mathrm{Ti}, \mathrm{NbN}, \mathrm{VO}_{x}, \mathrm{Nb}$, and $\mathrm{Al} / \mathrm{Nb}$ ), and Schottky barrier diodes (SBDs) (such as InGaAs, Er/InGaAlAs, and products from Virginia Diodes, 
Inc.) are in widespread use, they suffer from poor noise equivalent power (NEP) (only $10^{-9}-10^{-10} \mathrm{~W} \mathrm{~Hz}^{-1 / 2}$ level for Golay cells and pyroelectrics), slow response (ms level for Golay cells, pyroelectrics and uncooled semiconducting microbolometers), or narrow spectral bandwidth (multiple modules for SBDs to achieve broad spectral bandwidth) ${ }^{9}$.

In the past decades, much effort has been devoted to developing novel techniques for room temperature millimetre and terahertz wave direct detection. Complementary metal-oxide-semiconductor and plasmabased field electric transistor (FET), high-electronmobility transistor and metal-oxide-semiconductor field-effect transistor have experienced a development boom, including adoption of $\mathrm{Si}, \mathrm{SiGe}, \mathrm{GaAs} / \mathrm{AlGaAs}$, InGaAs, InGaP/InGaAs/GaAs, and GaN/AlGaN material systems ${ }^{10,11}$. These devices possess multiple electrodes and sub-micrometer scale channels to arouse photoresponse. Terahertz photoconductive antennas (PCs) are equipped with a local femtosecond laser to pump a highresistivity and ultrashort-carrier-lifetime semiconductor (such as GaAs, InGaAs, InGa(Al)As, InP, and GaAs nanowires $)^{12-18}$. Such technique is well adopted in terahertz time domain systems. Millimetre and terahertz wave detectors based on emerging materials ${ }^{19-33}$ such as black phosphorus, perovskites, carbon nanotubes, Dirac semimetals, topological insulators, particularly graphene, have also been demonstrated with plasmonic, hot electron, and photothermoelectric strategies and an NEP level of $10^{-11} \mathrm{~W} \mathrm{~Hz}^{-1 / 2}$ has been achieved ${ }^{34}$.

Millimetre and terahertz wave detection can also be realized based on nonequilibrium electrons induced by surface plasmon polaritons (SPPs) ${ }^{35}$. In this methodology, a high electron mobility plasmonic semiconductor, which possesses negative permittivity in millimetre and terahertz waves, is used in an antenna-assisted subwavelength ohmic metal-semiconductor-metal structure. Under illumination, the SPPs in such semiconductor would induce nonequilibrium electrons which form photocurrent/photovoltage under a voltage bias. In the previous work, the detection was verified by simulation and experiment from devices made of plasmonic InSb in millimetre and terahertz wave range. However, as InSb film was polished from bulk wafer and adhered to sapphire substrate, it is difficult for fabricating large format arrays. In addition, since the antenna is based on narrowband half-wave dipole antenna, it has limited coupling efficiency and is not suitable for broad spectral band detection.

Subwavelength nanostructures have demonstrated their excellent capability in controlling and modulating light-matter interactions ${ }^{36-40}$. However, current applications are mainly based on metals that possess plasma frequencies in visible or ultraviolet ranges of the electromagnetic spectrum. In millimetre and terahertz wave range, it is quite difficult to directly drive a strong light-matter interaction by using metallic nanostructures. Spoof SPPs from periodic holes in a metal surface is a choice to solve this issue ${ }^{41,42}$. Besides, owing to the relatively lower plasma frequency of the doped or narrow band gap semiconductors, they can be used as excellent plasmonic materials for longer-wavelength millimetre and terahertz waves ${ }^{43-50}$. The adoption of nanostructures based on plasmonic semiconductor in longer wavelength is of potential to boost multiple functionalities, for example, the photoelectrical conversion capability.

Here, we report direct detection of millimetre and terahertz waves based on epitaxially grown InSb/AlInSb/ $\mathrm{GaSb} / \mathrm{GaAs}$ by molecular-beam epitaxy (MBE). The InSb films in such a novel structure possess high electron mobility and negative permittivity in a broad millimetre and terahertz wave band, and further, it is suitable for fabrication of large format arrays. A broad spectral bandwidth planar equiangular spiral antenna is designed to efficiently couple millimetre and terahertz waves. A nanogroove array is fabricated in InSb layer, which can arouse strong excitation of millimetre and terahertz wave SPPs, especially at the InSb-air interfaces, leading to a general improvement of $50-100 \%$ for detection performance. An NEP of $2.2 \times 10^{-14} \mathrm{~W} \mathrm{~Hz}^{-1 / 2}$ or a detectivity (D) of $2.7 \times 10^{12} \mathrm{~cm} \mathrm{~Hz}^{1 / 2} \mathrm{~W}^{-1}$ is achieved at $1.75 \mathrm{~mm}$ $(0.171 \mathrm{THz})$ at room temperature. The device also shows a broad spectral band detection from $0.9 \mathrm{~mm}(0.330 \mathrm{THz})$ to $9.4 \mathrm{~mm}(0.032 \mathrm{THz})$ and a fast response speed of $3.5 \mu \mathrm{s}$. By moderately decreasing the temperature to the thermoelectric cooling of $200 \mathrm{~K}$, the corresponding NEP, $D$ ", and response speed can be further improved to $3.8 \times$ $10^{-15} \mathrm{~W} \mathrm{~Hz}^{-1 / 2}, 1.6 \times 10^{13} \mathrm{~cm} \mathrm{~Hz}^{1 / 2} \mathrm{~W}^{-1}$, and $780 \mathrm{~ns}$, respectively.

\section{Results}

Figure 1a shows the epitaxially grown InSb-based structure on GaAs used for making devices. It contains a GaSb buffer layer $(500 \mathrm{~nm})$, an $\mathrm{Al}_{1-x} \mathrm{In}_{x} \mathrm{Sb}$ graded layer $(1000 \mathrm{~nm})$, an $\mathrm{Al}_{0.15} \mathrm{In}_{0.85} \mathrm{Sb}$ barrier layer $(500 \mathrm{~nm})$ and an InSb active layer $(2000 \mathrm{~nm})$ which are sequentially grown on a GaAs substrate $(300 \mu \mathrm{m})$ by MBE. The measured room temperature electron concentration and mobility are $2.52 \times 10^{16} \mathrm{~cm}^{-3}$ and $4.95 \times 10^{4} \mathrm{~cm}^{2} \mathrm{~V}^{-1} \mathrm{~s}^{-1}$, respectively (Figs. S1 and S2). The permittivity of the InSb film in millimetre and terahertz wave range is negative in frequency up to $\sim 3 \mathrm{THz}$ or in wavelength down to $0.1 \mathrm{~mm}$ at room temperature according to Drude model (see "Materials and methods" and Fig. S3), which makes InSb is a good plasmonic semiconductor ${ }^{44-48}$. As such, millimetre and terahertz wave SPPs can be excited in InSb, which will induce the generation of nonequilibrium electrons $^{35,51,52}$. 
a

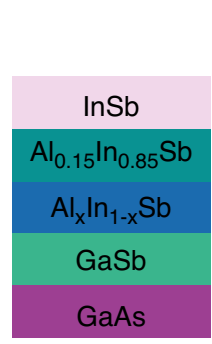

b

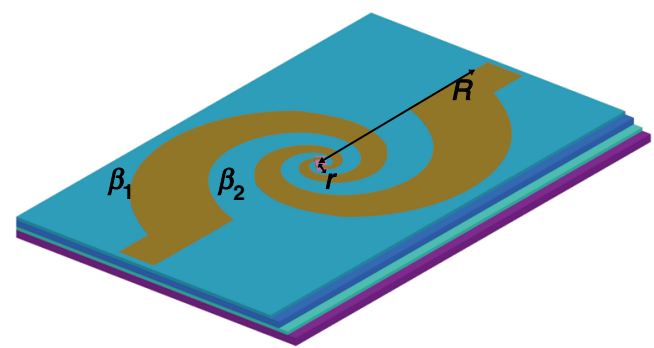

C

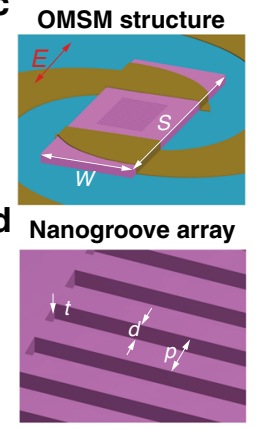

e $\operatorname{Max}$

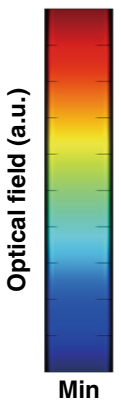

h

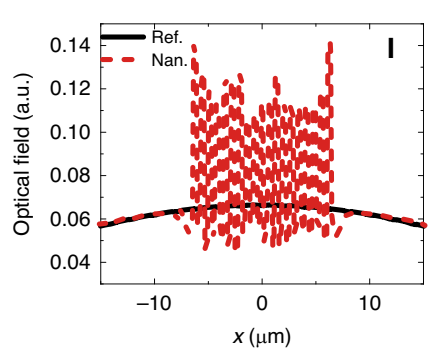

$\mathbf{f}$

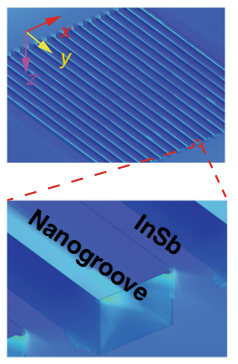

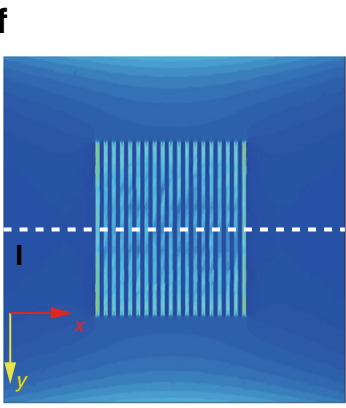

i

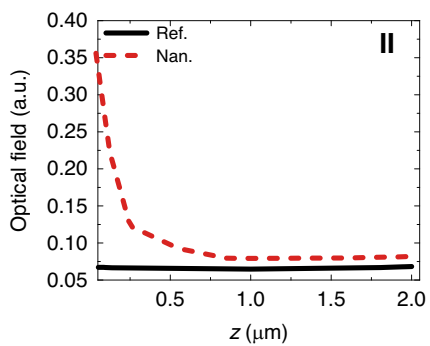

g Air InSb Air InSb Air
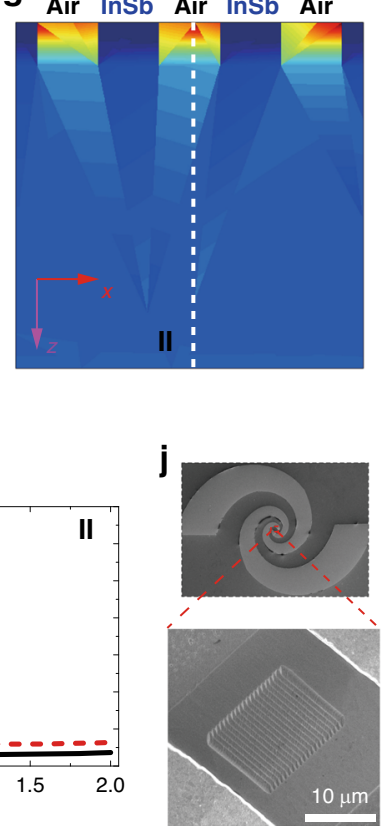

Fig. 1 InSb device design. a Structure of the epitaxially grown InSb on GaAs substrate. $\mathbf{b}$ Schematic of the spiral antenna-assisted device. $R$ and $r$ are respectively the out and inner radius of the antenna. $\beta_{1}$ and $\beta_{2}$ represent curves of the arm. $\mathbf{c}$ The central ohmic metal-semiconductor-metal (OMSM structure). $s(50 \mu \mathrm{m})$ and $w(30 \mu \mathrm{m})$ are the length and width of the mesa between ohmic contacts. $E$ denotes the TM polarization orientation (to arouse SPPs in nanogroove array) of incident electromagnetic waves. $\mathbf{d}$ Nanogroove array. The period $p$, width $d$, and depth $t$ of the nanogroove are 700, 350, and $250 \mathrm{~nm}$, respectively. e Distribution of simulated optical field for nanogroove InSb device at $0.171 \mathrm{THz}$. $\mathbf{f}$ Distribution of the field at $x y$ plane ( $z=250 \mathrm{~nm}$, bottom plane of the nanogroove array). $\mathbf{g}$ Distribution of the field at $x z(y=0)$ plane. Optical field distribution along cut-line I (h) and cut-line II (i) as denoted in $\mathbf{f}$ and $\mathbf{g}$, respectively. $\mathbf{j}$ Scanning electron microscopy image of the plasmonic nanogroove InSb device. The bottom panel is the zoom-in view of the nanogroove array area.

To obtain broad spectral band detection, a planar equiangular spiral antenna (see Fig. 1b) is designed to couple millimetre and terahertz waves. Such antenna can be described as ${ }^{53}$

$$
\beta_{1}=r e^{\alpha \phi} ; \beta_{2}=r e^{\alpha(\phi-\theta)}
$$

where $\beta_{1}$ and $\beta_{2}$ represent curves of the antenna's arm, $r$ is the initial inner radius, $\alpha$ is the growth factor, and $\theta$ is the rotation angle for the arm. This antenna includes two arms and the other arm can be determined by the same equations rotated by an angle of $180^{\circ}$. In our design, $\theta=$ $\pi / 2$ and $\alpha=0.33$. The operational lower and higher frequencies of such antenna are roughly given by

$$
f_{\text {low }}=\frac{c}{\sqrt{2} \pi R \sqrt{\varepsilon_{\mathrm{r}}+1}} ; f_{\text {high }}=\frac{c}{\sqrt{2} \pi r \sqrt{\varepsilon_{\mathrm{r}}+1}}
$$

where $R$ is the out radius of the antenna, $c$ is the speed of light in vacuum, and $\varepsilon_{\mathrm{r}}$ is the relative permittivity of the dielectric layer on which the antenna is deposited. In our design, $\varepsilon_{\mathrm{r}}$ is around 15 for AlInSb, $r=0.030 \mathrm{~mm}$, and $R=$ $0.560 \mathrm{~mm}$. Therefore, the operational frequency limit can be derived as $f_{\text {low }}=0.029 \mathrm{THz}$ and $f_{\text {high }}=0.450 \mathrm{THz}$. The 
broadband coupling capability of this antenna was also verified by CST software (Fig. S4). It has also demonstrated great coupling efficiency for millimetre and terahertz waves ${ }^{14,53}$.

A plasmonic semiconductor InSb nanogroove (or slot) array was integrated into the device (as shown in Fig. 1c, d). This kind of InSb periodic structure has been demonstrated with great capability to significantly enhance the optical field (or SPPs), especially at the $\mathrm{InSb}$-dielectric interface, in millimetre and terahertz wave range due to the plasmonic nature (or negative permittivity) of $\mathrm{InSb}^{44,47,54-56}$. These SPPs can transfer their energies to electrons or phonons in InSb, leading to absorption of incident millimetre and terahertz waves by the nanogroove array. The strong local field enhancement would induce more nonequilibrium electrons, which results in larger photocurrent/photovoltage and leading to increase in detection sensitivity ${ }^{35}$. As shown in Fig. 1d, the period $p$, width $d$, and depth $t$ of the nanogroove array are 700,350 , and $250 \mathrm{~nm}$, respectively. As such, the wavelength ( $\mathrm{mm}$ level) of electromagnetic wave is much longer than the period $(700 \mathrm{~nm})$ of this nanogroove array $(\lambda » p)$. Such deep subwavelength plasmonic nanogroove is expected to offer a strong excitation of SPPs.

In our design, the overall absorption of the device is first determined by the coupling of the antenna, which can be expressed as $e_{0}=e_{\mathrm{r}} \cdot e_{\mathrm{c}} \cdot e_{\mathrm{d}}$, where, $e_{0}$ is the total efficiency, $\mathrm{e}_{\mathrm{r}}$ is the reflection efficiency, $e_{\mathrm{c}}$ is the conduction efficiency, and $e_{d}$ is the dielectric efficiency. Usually, it is hard to determine the three components either from calculation or experiments ${ }^{53}$. Even if we can determine the exact coupling efficiency, it is only the first step in the whole absorption process for the device. After coupling, the incident millimetre and terahertz waves will generate SPPs owing to the negative permittivity of InSb. In this step, only part of incident waves can generate SPPs, which then transfer their energies to electrons in $\mathrm{InSb}$ and therefore induce nonequilibrium electrons to form photocurrent. In this step, both the conversion ratio from coupled incident waves to SPPs and from SPPs to nonequilibrium electrons are difficult to estimate. Besides, we need to consider the influence of free-electron absorption by the metallic antenna. All these factors make it hard to determine the transmission or absorption of the device.

To gain further insight on generation of the SPPs, we simulate the distribution of SPP intensity in reference and nanogroove array InSb devices and the results for nanogroove array $\mathrm{InSb}$ are presented in Fig. 1e-g. As shown in Fig. 1e, obvious field enhancement is indeed observed in nanogroove InSb array with its maximum at around the interface between InSb and Air. To have a better view, we extracted the field distribution in $x y(z=0$, surface of InSb) and $x z(y=0)$ planes for both devices (Fig. S5). As shown, InSb mesa with nanogrooves demonstrates large intensity of SPPs compared to reference one, that is, the nanogroove device will absorb more incident photons. As the SPPs in air (or within the nanogroove above the bottom surface) do not contribute to photoconductive carriers (or nonequilibrium electrons), we plot distributions of SPPs in $x y$ plane located at the bottom of the nanogroove array $(z=250 \mathrm{~nm})$ (Fig. 1f) and in $x z(y=0)$ plane (only a few periods in direction of $x$-axis for better view) (Fig. 1g). The InSb mesa with nanogrooves still exhibits obvious field enhancement, particularly at the interface between InSb and air in the nanogrooves, which leads to overall enhancement in the whole InSb mesa and therefore more absorption. Figure $1 \mathrm{~h}, \mathrm{i}$, respectively, plot the field distribution along cut lines I and II as denoted in Fig. 1f, g. It is observed that the distribution of SPPs is kind of uniform for reference device; however, after integration of a nanogroove array, it exhibits maximum at the nanogroove area and gradually decays into InSb. Such plasmonic behavior by InSb is very like its metallic counterparts in visible range. The field enhancement in nanogroove InSb array is supposed to improve the detection performance as more nonequilibrium electrons would be induced accompanied by more absorption of millimetre and terahertz waves. It is noted here that by varying dimension of the nanogroove, SPPs excitation would be varied ${ }^{55}$. In this work, we try to demonstrate the capability of such nanogroove array to improve detecting performance and do not study its size-effect on performance. In fabrication, two kinds of devices, one denoted as "Ref" without nanogroove structure, and the other one denoted as "Nan" with plasmonic nanogroove array were fabricated in the same chip. The fabrication process was discussed in detail in "Materials and methods". The scanning electron microscopy image of a typical nanogroove array device is shown in Fig. $1 \mathrm{j}$.

Dark current-voltage $(I-V)$ curves in Fig. 2a demonstrate good ohmic and symmetry characters of devices. The nanogroove and reference devices exhibit a comparable dark current level, indicating very weak effects of the shallow plasmonic nanogroove. For our devices, in addition to thermal Johnson-Nyquist noise $\left(v_{t}\right)$ in FET structures, the noise $\left(v_{\mathrm{b}}\right)$ due to voltage bias (dark current) should also be included. The total noise $\left(v_{\mathrm{n}}\right)$ can be described by by, $19,21,27,35^{2}$

$$
v_{\mathrm{n}}=\left(v_{\mathrm{t}}^{2}+v_{\mathrm{b}}^{2}\right)^{1 / 2}=\left(4 k_{\mathrm{B}} \operatorname{Tr}+2 q I_{\mathrm{d}} r^{2}\right)^{1 / 2}
$$

where $k_{\mathrm{B}}$ is Boltzmann's constant in joules per Kelvin, $T$ is the detector's absolute temperature in Kelvin, $r$ is the resistance value of the device in ohms $(\Omega), q$ is the elementary charge, and $I_{\mathrm{d}}$ is the dark current of the device. We also used a spectrum analyzer to evaluate noise level of the devices, a typical noise spectrum can be found in inset of Fig. 2b. As shown, $1 / f$ noise dominates in low- 


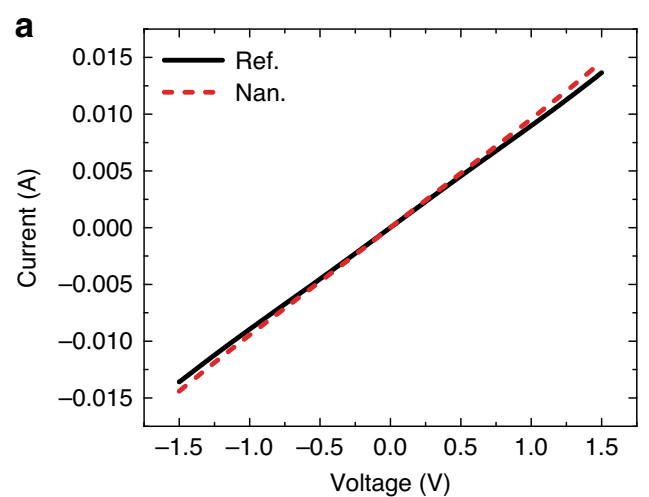

b

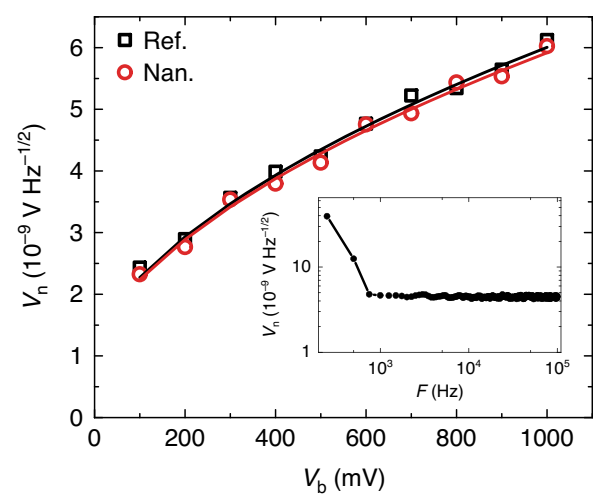

Fig. 2 Dark current and noise characterization. a Current-voltage characteristic curves of reference and nanogroove devices. b Typical calculated (lines) and measured (squares and circles) noise level of the nanogroove and reference devices. The inset is a typical measured noise spectrum.

frequency range below $1000 \mathrm{~Hz}$. Above $1000 \mathrm{~Hz}$, the noise becomes stable at a level of $10^{-9} \mathrm{~V} \mathrm{~Hz}^{1 / 2}$. Thus, the performance characterization was conducted under a modulation frequency of $10 \mathrm{kHz}$ to avoid such $1 / f$ disturbance. Figure $2 \mathrm{~b}$ plots the measured and calculated noise level of the devices under different voltage bias at $10 \mathrm{kHz}$. They agree well with each other and are comparable to other techniques at room temperature $^{25,57,58}$. The increment at higher bias is due to the rising of dark current shot noise $v_{\mathrm{b}}$. At $1000 \mathrm{mV}$, the noise level is $\sim 6 \mathrm{nV} \mathrm{Hz}^{-1 / 2}$.

The photoelectrical conversion capability of the devices is characterized by photoresponsivity $\left(R_{\mathrm{v}}\right), \mathrm{NEP}$, and $D$. They are defined $\mathrm{as}^{3}$

$$
R_{\mathrm{v}}=V /(p A) ; \mathrm{NEP}=v_{\mathrm{n}} / R_{\mathrm{v}}, D^{*}=\sqrt{A} / \mathrm{NEP}
$$

where $V$ is the photovoltage of the device, $p$ is the power density calibrated by a Golay cell and it has a typical value of $0.00171 \mathrm{~W} \mathrm{~m}^{-2}$ at $0.171 \mathrm{THz}, A$ is the effective absorption area of the detector, described as $A=G \lambda^{2}$ / $(4 \pi)$ if assuming the antenna is matched to its load ( $G$ is
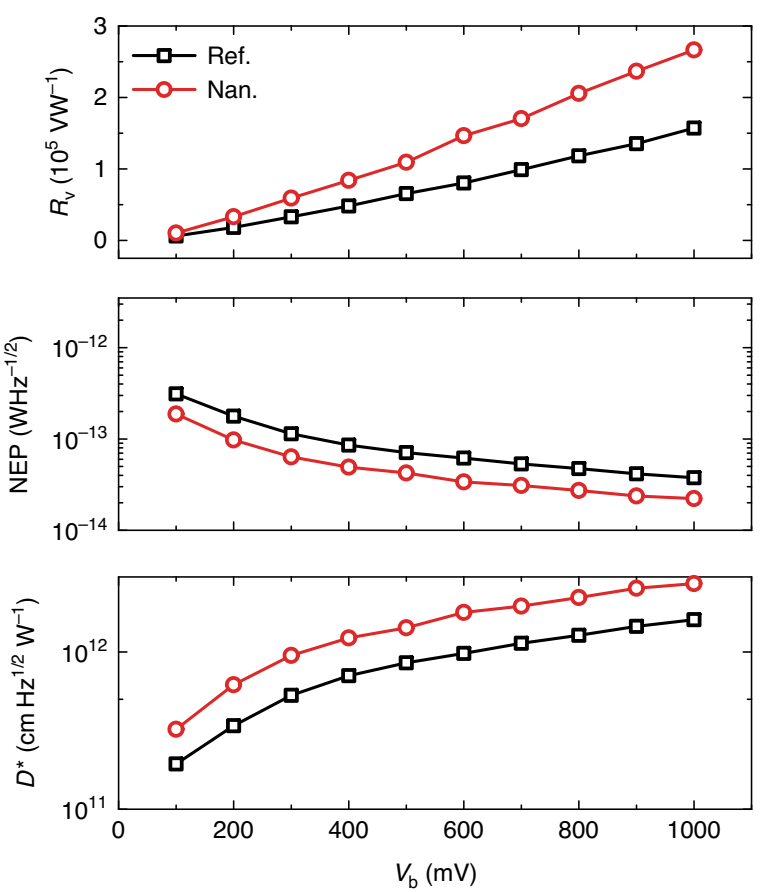

Fig. 3 Performance vs. bias. $R_{v}$ NEP, and $D^{*}$ of the devices at different voltage bias for room temperature operation at $0.171 \mathrm{THz}$.

the gain of the antenna and assumed to $G=1$ here. $)^{53}$, and $v_{\mathrm{n}}$ is the noise voltage as described in Eq. (3). Figure 3 shows the measured $R_{\mathrm{v}}, \mathrm{NEP}$, and $D^{*}$ of the two devices at different voltage bias $V_{\mathrm{b}}$ for room temperature operation at $1.75 \mathrm{~mm}(0.171 \mathrm{THz})$, where devices exhibit the best performance. Performance of the nanogroove device shows an overall $50-100 \%$ improvement compared to the reference one. $R_{\mathrm{v}}$ increases linearly with voltage bias as the collected nonequilibrium electrons is proportional to the drift velocity ${ }^{35}$. Under a voltage bias of $1000 \mathrm{mV}, R_{\mathrm{v}}$ of the reference and nanogroove devices are $1.57 \times 10^{5}$ and $2.66 \times 10^{5} \mathrm{VW}^{-1}$, respectively. Such high $R_{\mathrm{v}}$ is comparable to that of a Golay cell ${ }^{3,9}\left(1 \times 10^{5} \mathrm{~V} \mathrm{~W}^{-1}\right)$ and a dual-gated bilayer graphene hot electron bolometer $^{23}$ operated at $5 \mathrm{~K}\left(1 \times 10^{5} \mathrm{VW}^{-1}\right)$. It is much higher than other room temperature techniques ${ }^{3}$. For such millimetre and terahertz wave detector, due to the involvement of a couple antenna, we usually do not use quantum efficiency to evaluate its performance. For a photodiode, its quantum efficiency can be expressed as $\eta$ $=\left(I_{\mathrm{p}} / e\right) /(P / h v)=\left(I_{\mathrm{p}} / P\right) \times(h v / e)=R_{\mathrm{A}} \times(h v / e)^{3,8}$, where $I_{\mathrm{p}}$ is the photocurrent, $e$ is the element charge, $P$ is the incident power, $h v$ is the quantum energy of the incident photon, and $R_{\mathrm{A}}$ is the responsivity in $\mathrm{A} / \mathrm{W}$. The typical value of $\eta$ for a photodiode is less than $100 \%$. But for a photoconductive detector (there is no internal built-in field), its quantum efficiency is defined by the term photoconductive gain, which can be larger than 100\% 
when expressed in percentage. Here, we can define the quantum efficiency of our detector following that of a photodiode. At $0.171 \mathrm{THz}$ and $1000 \mathrm{mV}$ bias, the typical quantum efficiency of our nanogroove $\left(R_{\mathrm{v}}=2.66 \times 10^{5} \mathrm{~V}\right.$ $\left.\mathrm{W}^{-1}, R=105.7 \Omega\right)$ and reference $\left(R_{\mathrm{v}}=1.57 \times 10^{5} \mathrm{~V} \mathrm{~W}^{-1}\right.$, $R=111.7 \Omega$ ) devices are $176 \%$ and $98 \%$ respectively, or we say, the photoconductive gain are 1.76 and 0.98 , respectively. NEP and $D^{*}$ also show improvement while increasing bias, but due to increment of noise level (see Fig. 2), they tend to saturate at a relatively large bias of $1000 \mathrm{mV}$. At this point, NEP of the reference and nanogroove devices are as low as $3.7 \times 10^{-14}$ and $2.2 \times$ $10^{-14} \mathrm{~W} \mathrm{~Hz}^{-1 / 2}$, respectively, demonstrating a $1-2$ order of magnitude improvement compared to commercially available zero-bias SBD $\left(10^{-12} \mathrm{~W} \mathrm{~Hz}^{-1 / 2}\right.$ level $\left.^{59}\right)$ (performance of other uncooled techniques has been summarized in refs. ${ }^{3,35}$ and Table S1). The corresponding $D^{*}$ reaches to $1.6 \times 10^{12}$ and $2.7 \times 10^{12} \mathrm{~cm} \mathrm{~Hz}^{1 / 2} \mathrm{~W}^{-1}$, which are two orders of magnitude higher than that of an ideal thermal-type detector ${ }^{3}\left(\sim 1.9 \times 10^{10} \mathrm{~cm} \mathrm{~Hz}^{1 / 2} \mathrm{~W}^{-1}\right.$ level if assuming $2 \pi$ FOV@300 K).

Spectral response was characterized in a broad frequency range from 0.032 to $0.330 \mathrm{THz}$ limited by our facility (Fig. 4). The corresponding quantum efficiency is also derived as shown in Fig. S6. The experimental process is discussed in greater detail in "Materials and methods". Both devices demonstrate good photoresponse in four frequency bands (0.03-0.04, 0.075-0.110, 0.150-0.220, and 0.225-0.330 THz).

Such broad spectral bandwidth nature can be partially ascribed to the broadband coupling capability of the spiral antenna ${ }^{14,53}$, which is much more efficient than the narrowband dipole-like antenna adopted in the previous report $^{35}$. In addition, negative permittivity of InSb over a broad millimetre and terahertz wave band (up to $\sim 3 \mathrm{THz}$ ) allows efficient generation of localized SPPs (Fig. S3), and therefore, broad spectral bandwidth photoresponse. The multiple peak response character is caused by the multiple internal reflection from the substrate interface (or excitation of substrate modes), which can lead to antenna pattern (gain and input impedance) degradation ${ }^{60}$. This phenomenon can be solved by making substrate thinner, using interconnection metallization, integrating a substrate lenses, or using a conductive substrate ${ }^{61-63}$. The difference for peak performance in the four bands may originate from the difference in coupling efficiency of the antenna. As shown in Fig. S4, the antenna can couple the maximum optical field (largest power intensity) into the device at $0.171 \mathrm{THz}$, then sequentially decreased at $0.287,0.094$, and $0.037 \mathrm{THz}$. It may also be related to conversion efficiency from coupled light to SPPs and from SPPs to nonequilibrium electrons.

In Fig. $4 \mathrm{a}$, b, we plot the typical $R_{\mathrm{v}}\left(\sim 2000 \mathrm{VW}^{-1}\right)$ and $\mathrm{NEP}\left(\sim 1 \times 10^{-12} \mathrm{~W} \mathrm{~Hz}^{-1 / 2}\right)$ of state-of-the-art zero-bias diodes (ZBDs) in corresponding frequency bands (ZBD module is not available for $0.03-0.04 \mathrm{THz}$ bands and $D^{*}$ is a

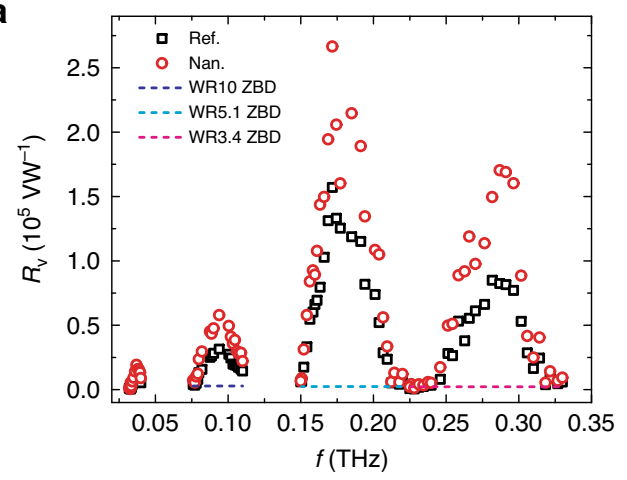

b

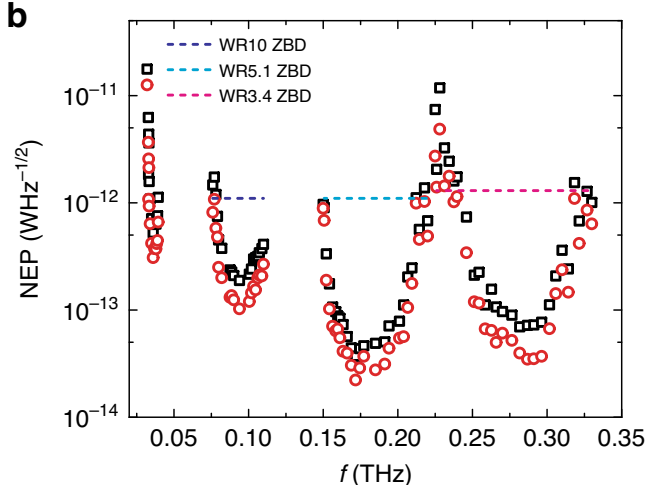

C

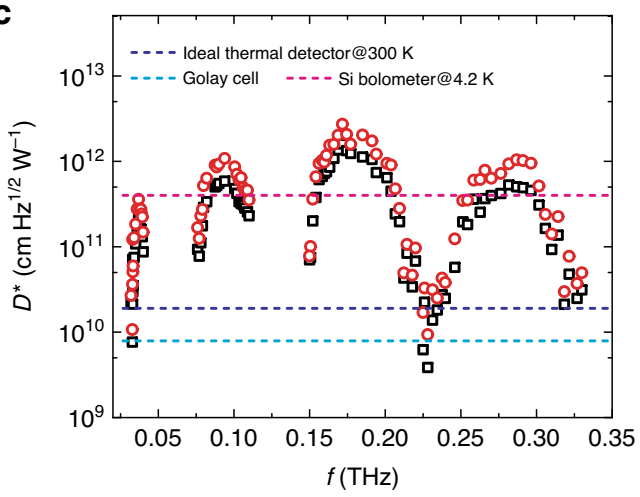

Fig. 4 Spectral response. $R_{\mathrm{v}}(\mathbf{a}), \operatorname{NEP}(\mathbf{b})$, and $D^{*}$ (c) of the devices in a frequency range of $0.032-0.330 \mathrm{THz}$ under a voltage bias of $1000 \mathrm{mV}$. The dash lines in $\mathbf{a}$ and $\mathbf{b}$ represent typical performance of state-ofthe-art commercial zero-bias SBDs (ZBDs) from VDI for corresponding frequency band ${ }^{59}$. The dash lines in $\mathbf{c}$ represent the ideal $D^{*}$ of a thermal-type detector at room temperature, Golay cell and an Si bolometer at $4.2 \mathrm{~K}$.

not used as a figure of merit for ZBDs). As shown, our devices demonstrate a general 1-2 orders of magnitude improvement in performance in a broadband range. With a single device, we achieve broad spectral bandwidth detection, which, in contrast, requires multiple modules for ZBD $^{57}$ (WR10, WR5.1, and WR3.4 as denoted in Fig. 4). In Fig. 4c, we plot $D^{*}$ of an ideal thermal detector ${ }^{3}$, a Golay cell (room temperature operation), and an $\mathrm{Si}$ bolometer 
(4.2 K). Our nanogroove device exhibits a 1-2 orders of magnitude improvement compared to Golay cell. $D$ is also higher than that of an ideal thermal-type detector at room temperature and it is even higher than that of the Si bolometer $(4.2 \mathrm{~K})$ in the frequency bands of $0.080-0.100$, $0.155-0.204$, and $0.266-0.300 \mathrm{THz}$. Specifically, at $0.287 \mathrm{THz}(1.05 \mathrm{~mm}), R_{\mathrm{v}}$ of our reference and nanogroove devices are respectively $8.2 \times 10^{4}$ and $1.7 \times 10^{5} \mathrm{~V} \mathrm{~W}^{-1}$. The measured NEP are $7.2 \times 10^{-14}$ and $3.5 \times 10^{-14} \mathrm{~W} \mathrm{~Hz}^{-1 / 2}$, respectively. $D^{\prime \prime}$ still shows a level of $10^{12} \mathrm{~cm} \mathrm{~Hz}^{1 / 2} \mathrm{~W}^{-1}$ for both. The NEPs in this frequency band are comparable to that of the plasmonic InAlAs/InGaAs/InP detector, which achieved a NEP of $4.8 \times 10^{-13} \mathrm{~W} \mathrm{~Hz}^{-1 / 2}$ at $0.2 \mathrm{THz}^{64}$. The performance decrease in low-frequency bands. For example, at $0.094 \mathrm{THz}(3.19 \mathrm{~mm})$, we achieve a $R_{\mathrm{v}}, \mathrm{NEP}$, and $D^{*}$ of $5.8 \times 10^{4} \mathrm{~V} \mathrm{~W}^{-1}, 1.0 \times 10^{-13} \mathrm{~W} \mathrm{~Hz}^{-1 / 2}$, and $1.1 \times 10^{12}$ $\mathrm{cm} \mathrm{Hz}^{1 / 2} \mathrm{~W}^{-1}$, respectively, which is comparable to the InAs/AlSb/AlGaSb heterojunction backward diode (NEP $=1.8 \times 10^{-13} \mathrm{~W} \mathrm{~Hz}^{-1 / 2}$ ) at the same frequency ${ }^{65}$ and the $\mathrm{AlGaN} / \mathrm{GaN}$ high electron mobility transistor at $0.14 \mathrm{THz}$ $\left(5.8 \times 10^{-13} \mathrm{~W} \mathrm{~Hz}^{-1 / 2}\right)^{66}$. Even at $0.037 \mathrm{THz}(8.11 \mathrm{~mm})$, the $R_{\mathrm{v}}$, NEP, and $D^{*}$ of the nanogroove device can reach to $1.9 \times 10^{4} \mathrm{VW}^{-1}, 3.1 \times 10^{-13} \mathrm{~W} \mathrm{~Hz}^{-1 / 2}$, and $3.6 \times 10^{11} \mathrm{~cm}$ $\mathrm{Hz}^{1 / 2} \mathrm{~W}^{-1}$, respectively. It is noted here that as the negative permittivity of InSb can hold until $\sim 3 \mathrm{THz}$ (Fig. S3), our InSb devices are promising for even higher frequency detection by engineering the coupling antenna, for example, by reducing the inner radius.

Temperature dependence of performance was characterized from room temperature (293 K) to thermoelectric cooling available up to $200 \mathrm{~K}$ (Fig. 5). As shown, the performance shows an overall improvement while decreasing the temperature. At $200 \mathrm{~K}, R_{\mathrm{v}}, \mathrm{NEP}$, and $D^{*}$ of the nanogroove device are $2.5 \times 10^{6} \mathrm{~V} \mathrm{~W}^{-1}, 3.8 \times 10^{-15} \mathrm{~W} \mathrm{~Hz}^{-1 / 2}$, and $1.6 \times 10^{13} \mathrm{~cm} \mathrm{~Hz}^{1 / 2} \mathrm{~W}^{-1}$, respectively, demonstrating a 5-6-fold enhancement compared to those at room temperature. Such performance improvement can be mainly ascribed to the increase of electron mobility at low temperatures $^{35,67}$ (Fig. S2). We do not study the performance of our devices at extremely low temperatures as our interests lay on uncooled or cheap thermoelectric cooling technology-based applications.

To gain an insight on response speed, we measured photoresponse of devices with respect to modulation rate of incident wave at $0.171 \mathrm{THz}$ under a bias of $1000 \mathrm{mV}$ (Fig. 6). Both devices demonstrate a $3 \mathrm{~dB}$ bandwidth of $10^{5} \mathrm{~Hz}$ for room temperature operation, corresponding to a rise time (signal from 0 to 63.2 of the maxima) of $3.5 \mu \mathrm{s}$ according to $t_{\mathrm{r}}=0.35 / f_{-3 \mathrm{~dB}}$, which is much faster than commercial Golay, pyroelectric and uncooled semiconducting microbolometer detectors $(\mathrm{ms} \mathrm{level})^{5}$. It is slower than metallic microbolometers ${ }^{68-71}$, which possess a response speed faster than $\sim 3 \mu$ s. It is also much slower than that of ZBDs, which can respond as fast as
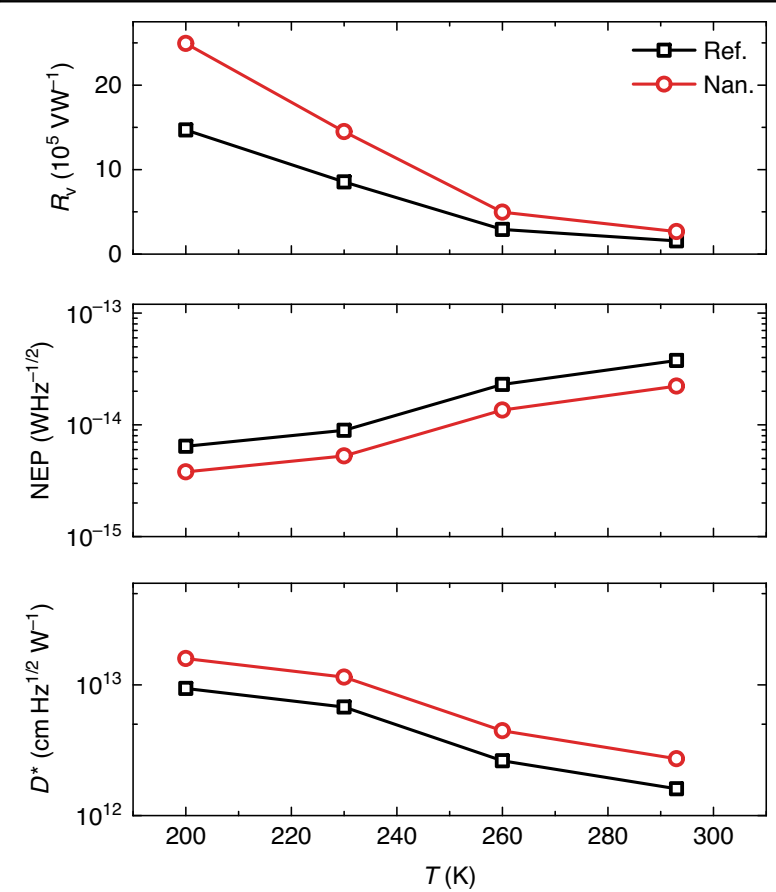

Fig. 5 Performance vs. temperature. $R_{v}$ NEP, and $D^{*}$ of the devices at different temperature under a voltage bias of $1000 \mathrm{mV}$ at $0.171 \mathrm{THz}$.

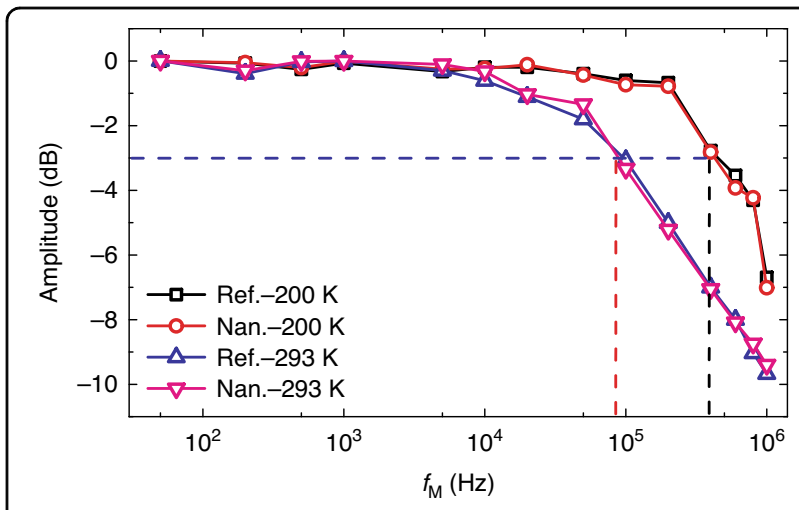

Fig. 6 Response bandwidth. Amplitude-frequency response of the devices at 293 and $200 \mathrm{~K}$.

nanosecond level (GHz bandwidth) ${ }^{59}$. Nevertheless, it can still meet the requirement of real-time imaging applicaions. At $200 \mathrm{~K}$, the corresponding $3 \mathrm{~dB}$ bandwidth and rise time are $4.5 \times 10^{5} \mathrm{~Hz}$ and $780 \mathrm{~ns}$, respectively, owing to the fast transition of nonequilibrium electrons at low temperature. In experiments, we observed very close response speed at other radiation frequencies. These devices operate on single type carrier mode (electrons). The detection is based on the undirectional flow of nonequilibrium electrons induced by SPPs. The lifetime of the nonequilibrium electrons here is similar to that of hot electrons in bulk InSb, which is around $50 \mathrm{~ns}$ at room 
temperature ${ }^{72,73}$. The response speed of our devices are probably governed by the decay time of SPPs, the lifetime of those nonequilibrium electrons, the time for photoconductive carriers to be collected by electrodes, and the RC factor in the whole measurement set-up ${ }^{35}$. Further study needs to be conducted to figure this out. In experiments, we also found that the photovoltage of the device is proportional to the power of radiation. As shown in Fig. S7, the linear dynamic range is derived as $21.7 \mathrm{~dB}$ at $0.171 \mathrm{THz}$ (limited by the maximum output power of the source).

\section{Discussion}

In conclusion, we have demonstrated sensitive and broadband millimetre and terahertz wave photodetectors that are based on epitaxially grown InSb/AlInSb/GaSb/ GaAs. A plasmonic nanogroove array is fabricated in the device to drive an overall $50-100 \%$ performance improvement. At room temperature, the device achieves a NEP of $2.2 \times 10^{-14} \mathrm{~W} \mathrm{~Hz}^{-1 / 2}$ and a $D^{\prime \prime}$ of $2.7 \times 10^{12} \mathrm{~cm}$ $\mathrm{Hz}^{1 / 2} \mathrm{~W}^{-1}$ at $1.75 \mathrm{~mm}$ under an applied bias of $1000 \mathrm{mV}$. The corresponding NEP and $D^{*}$ can be further improved to $3.8 \times 10^{-15}$ and $1.6 \times 10^{13} \mathrm{~cm} \mathrm{~Hz}^{1 / 2} \mathrm{~W}^{-1}$ while operating at $200 \mathrm{~K}$. The single plasmonic device achieves broad spectral bandwidth from $0.9 \mathrm{~mm}(0.330 \mathrm{THz})$ to $9.4 \mathrm{~mm}$ $(0.032 \mathrm{THz})$. Fast response speed of $3.5 \mu \mathrm{s}$ and $780 \mathrm{~ns}$ are, respectively, achieved at room temperature and $200 \mathrm{~K}$. Such high-performance photodetector would be a strong building block in wide applications of millimetre and terahertz waves. The integration of plasmonic semiconductor nanostructures is promising for developing novel and high-performance millimetre and terahertz wave optoelectrical devices.

\section{Materials and methods Device simulation}

In simulation, CST software was used to evaluate the coupling efficiency of the antenna. The typical coupling for four frequencies is presented in Fig. S4 (see Supporting Information). COMSOL Multiphysics was used to calculate the SPP generation in the plasmonic InSb nanogroove array. The frequency dependence of relative permittivity of InSb in millimetre and terahertz wave range can be described as $\varepsilon(\omega)=\varepsilon(0)-\omega_{\mathrm{p}}{ }^{2} /\left(\omega^{2}+\right.$ $\left.i \omega \tau^{-1}\right)^{45,55,56}(\varepsilon(0)$ is the static dielectric constant and is 16.8 for InSb, $\omega_{\mathrm{p}}$ is the plasma frequency, and $\tau$ is the relaxation time of the electrons). $\omega_{\mathrm{p}}$ is determined by $\omega_{\mathrm{p}}{ }^{2}$ $=q^{2} n /\left(m^{\prime \prime} \varepsilon_{0}\right)$, where $q$ is the elementary charge, $n$ is the electron density, $m^{\prime \prime}$ is the electron effective mass at the bottom of the band edge and is $0.014 m_{0}$ for InSb, and $\varepsilon_{0}$ is the permittivity in vacuum. The mobility of the carriers is related to the relaxation time by $\mu=q \tau / \mathrm{m}^{*}$. The calculated relative permittivity of $\mathrm{InSb}$ in frequency of interest is presented in Fig. S3.

\section{Device fabrication}

$\mathrm{SiO}_{2}(150 \mathrm{~nm})$ was first deposited on $\mathrm{InSb}$ epitaxial wafer, which was then patterned with electron-beam lithography using a negative resist ZEP. The patterned features were first etched with RIE to define $\mathrm{SiO}_{2}$ hard mask. The wafer was then etched by ICP-RIE to define the plasmonic InSb nanogroove array after resist removal by PR Asher. Before UV lithography to define InSb mesa, residual $\mathrm{SiO}_{2}$ was removed by BOE solution. The mesa etch was conducted based on chemical solution (HF: $\mathrm{H}_{2} \mathrm{O}_{2}$ :citric acid:DI $\mathrm{H}_{2} \mathrm{O}$ ). Next, metallic contacts and antennas $(300 \mathrm{~nm} \mathrm{Au}$ followed by $20 \mathrm{~nm}$ Ti adhesion layer) were defined via photolithography, e-beam evaporation, and a standard lift-off process. Multiple reference and nanogroove devices are processed in the same chip.

\section{Device characterization}

Dark current-voltage characteristics were measured under blackout conditions with a Keithley 2450 source meter. Noise spectra were obtained by a single channel $100 \mathrm{kHz}$ SR770 FFT spectrum analyzer. Millimetre and terahertz wave sources are based on commercially available VDI tenable synthesizer $(0.008-0.020 \mathrm{THz})$, WR28SGX (signal generator extension module 0.026-0.040 THz), WR10SGX-M (mini signal generator extension module $0.075-0.110 \mathrm{THz}), \quad$ WR $5.1 \times 2$ broadband Doubler $(0.150-0.220 \mathrm{THz}), \quad$ WR $3.4 \times 3$ broadband Tripler $(0.225-0.330 \mathrm{THz})$, and a PIN switch for fast modulation of output. Four horn antennas used for four millimetre and terahertz wave bands are WR10CH conical horn antenna $(0.075-0.110 \mathrm{THz})$, WR5.1CH conical horn antenna $(0.150-0.220 \mathrm{THz})$, WR3.4DH diagonal horn antenna $(0.225-0.330 \mathrm{THz})$, and WR28CH conical horn antenna (0.026-0.040 THz). The devices were mounted in a low-temperature dewar and biased by a direct current. The photovoltage output was collected by a lock-in amplifier after a preamplifier. The output radiation power from horn antennas was calibrated by a thermal Golay cell. The typical power density of the source at $0.171 \mathrm{THz}$ is $0.00171 \mathrm{~W} \mathrm{~m}^{-2}$.

\section{Acknowledgements}

J.T. acknowledges support from Nanyang Technological University Presidential Postdoctoral Fellowship. The work is also supported by Ministry of Education (grant no. 2017-T1-002-117 and RG 177/17) and A*Star (grant no. SERC

A1883c0002 and SERC 1720700038), Singapore. Z.H. acknowledges support from the China National Science Fund for Distinguished Young Scholars (61625505), Chinese Academy of Sciences (ZDBS-LY- JSC025), and Sino-Russia International Joint Laboratory (18590750500). The device fabrication was carried out at Nanyang NanoFabrication Centre (N2FC). J.T. thanks the help from Dr. Chong Gang Yih, Ms. Ngo Ling Ling, Dr. Yongquan Zeng, Dr. Lin Wu, Dr. Wei Zhou, Dr. Yiming Yin, and Dr. Tao Hu.

\section{Author contributions}

D.H.Z. supervised the project. J.T. is the principal investigator of the project, did device simulation, fabrication, and characterization. F.S. and J.T. fabricated the 
nanogroove array. T.Z. and J.T. drew the schematic. J.T. prepared the manuscript which was finalized by D.H.Z. and J.T. with input from all authors.

\section{Conflict of interest}

The authors declare no competing interests.

Supplementary information The online version contains supplementary material available at https://doi.org/10.1038/s41377-021-00505-w.

Received: 1 November 2020 Revised: 24 February 2021 Accepted: 1 March 2021

Published online: 15 March 2021

\section{References}

1. Ferguson, B. \& Zhang, X. C. Materials for terahertz science and technology. Nat. Mater. 1, 26-33 (2002)

2. Lewis, R. A. A review of terahertz detectors. J. Phys. D Appl. Phys. 52, 433001 (2019).

3. Rogalski, A. Infrared and Terahertz Detectors. 3rd edn (CRC Press, 2019).

4. Yang, Y. H. et al. Terahertz topological photonics for on-chip communication. Nat. Photonics 14, 446-451 (2020).

5. Chen, H. T. et al. Active terahertz metamaterial devices. Nature 444, 597-600 (2006).

6. Ahmadivand, A. et al. Terahertz plasmonics: the rise of toroidal metadevices towards immunobiosensings. Mater. Today 32, 108-130 (2020).

7. Jeong, Y. G., Bahk, Y. M. \& Kim, D. S. Dynamic terahertz plasmonics enabled by phase-change materials. Adv. Opt. Mater. 8, 1900548 (2020).

8. Ahmadivand, A. et al. Toroidal metaphotonics and metadevices. Laser Photonics Rev. 14, 1900326 (2020).

9. Sizov, F. Terahertz radiation detectors: the state-of-the-art. Semicond. Sci. Technol. 33, 123001 (2018).

10. Mittleman, D. Sensing with Terahertz Radiation (Springer, 2003).

11. Dyakonov, M. \& Shur, M. Shallow water analogy for a ballistic field effect transistor: new mechanism of plasma wave generation by dc current. Phys. Rev. Lett. 71, 2465-2468 (1993).

12. Peng, K. et al. Single nanowire photoconductive terahertz detectors. Nano Lett. 15, 206-210 (2015).

13. Peng, $\mathrm{K}$. et al. Three-dimensional cross-nanowire networks recover full terahertz state. Science 368, 510-513 (2020).

14. Wang, N. et al. Room-temperature heterodyne terahertz detection with quantum-level sensitivity. Nat. Astron. 3, 977-982 (2019).

15. Berry, C. W. et al. Significant performance enhancement in photoconductive terahertz optoelectronics by incorporating plasmonic contact electrodes. Nat. Commun. 4, 1622 (2013).

16. Burford, N. M. \& El-Shenawee, M. O. Review of terahertz photoconductive antenna technology. Opt. Eng. 56, 010901 (2017).

17. Siday, T. et al. Terahertz detection with perfectly-absorbing photoconductive metasurface. Nano Lett. 19, 2888-2896 (2019).

18. Castro-Camus, E. et al. Polarization-sensitive terahertz detection by multicontact photoconductive receivers. Appl. Phys. Lett. 86, 254102 (2005).

19. Vicarelli, L. et al. Graphene field-effect transistors as room-temperature terahertz detectors. Nat. Mater. 11, 865-871 (2012).

20. Bandurin, D. A. et al. Resonant terahertz detection using graphene plasmons. Nat. Commun. 9, 5392 (2018).

21. Viti, L. et al. Black phosphorus terahertz photodetectors. Adv. Mater. 27, 5567-5572 (2015)

22. Koppens, F. H. L. et al. Photodetectors based on graphene, other twodimensional materials and hybrid systems. Nat. Nanotechnol. 9, 780-793 (2014).

23. Yan, J. et al. Dual-gated bilayer graphene hot-electron bolometer. Nat. Nanotechnol. 7, 472-478 (2012).

24. Wei, J. et al. Ultrasensitive hot-electron nanobolometers for terahertz astrophysics. Nat. Nanotechnol. 3, 496-500 (2008)

25. Cai, X. H. et al. Sensitive room-temperature terahertz detection via the photothermoelectric effect in graphene. Nat. Nanotechnol. 9, 814-819 (2014).

26. Komiyama, S. et al. A single-photon detector in the far-infrared range. Nature 403, 405-407 (2000).
27. Li, Y. F. et al. Ultrabroadband, ultraviolet to Terahertz, and high sensitivity $\mathrm{CH}_{3} \mathrm{NH}_{3} \mathrm{Pbl}_{3}$ perovskite photodetectors. Nano Lett. 20, 5646-5654 (2020).

28. Chen, S. L. et al. Efficient real-time detection of terahertz pulse radiation based on photoacoustic conversion by carbon nanotube nanocomposite. Nat. Photonics 8, 537-542 (2014).

29. Mittendorff, M. et al. Optical gating of black phosphorus for terahertz detection. Nano Lett. 17, 5811-5816 (2017).

30. Viti, L. et al. Plasma-wave terahertz detection mediated by topological insulators surface states. Nano Lett. 16, 80-87 (2016).

31. Liu, Y. et al. High-performance, ultra-broadband, ultraviolet to terahertz photodetectors based on suspended carbon nanotube films. ACS Appl. Mater. Interfaces 10, 36304-36311 (2018).

32. Wang, Y. X. et al. Ultrabroadband, sensitive, and fast photodetection with needle-like $\mathrm{EuBiSe}_{3}$ single crystal. ACS Photonics 6, 895-903 (2019).

33. Deng, T. et al. Three-dimensional graphene field-effect transistors as highperformance photodetectors. Nano Lett. 19, 1494-1503 (2019).

34. Rogalski, A., Kopytko, M. \& Martyniuk, P. Two-dimensional infrared and terahertz detectors: outlook and status. Appl. Phys. Rev. 6, 021316 (2019).

35. Tong, J. C. et al. Surface plasmon induced direct detection of long wavelength photons. Nat. Commun. 8, 1660 (2017).

36. Zijlstra, P., Chon, J. W. M. \& Gu, M. Five-dimensional optical recording mediated by surface plasmons in gold nanorods. Nature 459, 410-413 (2009).

37. Luo, X. G. et al. Extraordinary optical fields in nanostructures: from subdiffraction-limited optics to sensing and energy conversion. Chem. Soc. Rev. 48, 2458-2494 (2019).

38. Lin, R. J. et al. Achromatic metalens array for full-colour light-field imaging. Nat. Nanotechnol. 14, 227-231 (2019).

39. Garcia-Vidal, F. J. et al. Light passing through subwavelength apertures. Rev. Mod. Phys. 82, 729-787 (2010).

40. Genet, C. \& Ebbesen, T. W. Light in tiny holes. Nature 445, 39-46 (2007).

41. Pendry, J. B., Martin-Moreno, L. \& Garcia-Vidal, F. J. Mimicking surface plasmons with structured surfaces. Science 305, 847-848 (2004).

42. Shen, X. P. \& Cui, T. J. Ultrathin plasmonic metamaterial for spoof localized surface plasmons. Laser Photonics Rev. 8, 137-145 (2014).

43. Kumar, G. et al. Terahertz surface plasmon waveguide based on a onedimensional array of silicon pillars. N. J. Phys. 15, 085031 (2013).

44. Morgado, T. A. et al. Ultraconfined interlaced plasmons. Phys. Rev. Lett. 107, 063903 (2011).

45. Giannini, V. et al. Scattering efficiency and near field enhancement of active semiconductor plasmonic antennas at terahertz frequencies. Opt. Express 18, 2797-2807 (2010).

46. Wang, X. et al. Interference-induced terahertz transparency in a semiconductor magneto-plasma. Nat. Phys. 6, 126-130 (2010).

47. Deng, L. Y. et al. Direct optical tuning of the terahertz plasmonic response of InSb subwavelength gratings. Adv. Opt. Mater. 1, 128-132 (2013).

48. Hanham, S. M. et al. Broadband terahertz plasmonic response of touching InSb disks. Adv. Mater. 24, OP226-OP230 (2012).

49. Agrawal, A. et al. Localized surface plasmon resonance in semiconductor nanocrystals. Chem. Rev. 118, 3121-3207 (2018).

50. Rivas, J. G. et al. Propagation of surface plasmon polaritons on semiconductor gratings. Phys. Rev. Lett. 93, 256804 (2004).

51. Sundararaman, R. et al. Theoretical predictions for hot-carrier generation from surface plasmon decay. Nat. Commun. 5, 5788 (2014).

52. Clavero, C. Plasmon-induced hot-electron generation at nanoparticle/metaloxide interfaces for photovoltaic and photocatalytic devices. Nat. Photonics $\mathbf{8}$, 95-103 (2014).

53. Balanis, C. A. Antenna Theory: Analysis and Design 4th edn (Wiley, 2016).

54. Azad, A. K. et al. A review of terahertz plasmonics in subwavelength holes on conducting films. IEEE J. Sel. Top. Quantum Electron. 19, 8400416 (2013).

55. Parthasarathy, R. et al. Enhanced coupling of subterahertz radiation with semiconductor periodic slot arrays. Phys. Rev. Lett. 98, 153906 (2007).

56. Rivas, J. G. et al. Transmission of $\mathrm{THz}$ radiation through $\mathrm{InSb}$ gratings of subwavelength apertures. Opt. Express 13, 847-859 (2005).

57. Vitiello, M. S. et al. Room-temperature terahertz detectors based on semiconductor nanowire field-effect transistors. Nano Lett. 12, 96-101 (2012).

58. Qin, H. et al. Heterodyne detection at 216, 432, and $648 \mathrm{GHz}$ based on bilayer graphene field-effect transistor with quasi-optical coupling. Carbon 121, 235-241 (2017).

59. VDI ZBDs. https://www.vadiodes.com/en/products/detectors (2020).

60. Pozar, D. Considerations for millimeter wave printed antennas. IEEE Trans. Antennas Propag. 31, 740-747 (1983). 
61. Dhiflaoui, A. et al. Full wave numerical analysis of wideband and high directive log spiral THz photoconductive antenna. Int. J. Numer. Model. Electron. Netw. Devices Fields 33, e2761 (2020).

62. Sakhno, M., Gumenjuk-Sichevska, J. \& Sizov, F. Modeling of the substrate influence on multielement THz detector operation. J. Infrared Millim. Terahertz Waves 35, 703-719 (2014).

63. Nguyen, T. K. et al. Numerical study of self-complementary antenna characteristics on substrate lenses at terahertz frequency. J. Infrared Millim. Terahertz Waves 33, 1123-1137 (2012).

64. Kurita, Y. et al. Ultrahigh sensitive sub-terahertz detection by InP-based asymmetric dual-grating-gate high-electron-mobility transistors and their broadband characteristics. Appl. Phys. Lett. 104, 251114 (2014).

65. Zhang, Z. et al. Sub-micron area heterojunction backward diode millimeterwave detectors with $0.18 \mathrm{pW} / \mathrm{Hz}^{1 / 2}$ noise equivalent power. IEEE Microw. Wirel. Components Lett. 21, 267-269 (2011).

66. Hou, H. W. et al. High temperature terahertz detectors realized by a GaN high electron mobility transistor. Sci. Rep. 7, 46664 (2017).
67. Sze, S. M. \& Ng, K. K. Physics of Semiconductor Devices 3rd edn (Wiley, 2006).

68. Grossman, E. N. \& Miller, A. J. Active millimeter-wave imaging for concealed weapons detection. In Proceedings of SPIE 5077, Passive Millimeter-Wave Imaging Technology VI and Radar Sensor Technology VII. SPIE, Orlando, 62-70 (2003).

69. Tu, X. C. et al. $\mathrm{Nb}_{5} \mathrm{~N}_{6}$ microbolometer arrays for terahertz detection. Chin. Phys. B 22, 040701 (2013)

70. Kašalynas, I. et al. Spectroscopic terahertz imaging at room temperature employing microbolometer terahertz sensors and its application to the study of carcinoma tissues. Sensors 16, 432 (2016).

71. Rutledge, D. \& Schwarz, S. Planar multimode detector arrays for infrared and millimeter-wave applications. IEEE J. Quantum Electron. 17, 407-414 (1981).

72. Tanimura, H., Kanasaki, J. \& Tanimura, K. Ultrafast scattering processes of hot electrons in InSb studied by time- and angle-resolved photoemission spectroscopy. Phys. Rev. B 91, 045201 (2015).

73. Shur, M. Handbook Series on Semiconductor Parameters (World Scientific, 1996). 